\title{
Toxico-Informatics: Study of Natural Ligand for New Disease Era
}

\author{
Vijay Laxmi Saxena ${ }^{1}$, Shrasti Gupta ${ }^{2}$, Priya Verma ${ }^{3}$ \\ ${ }^{1}$ Bioinformatics Infrastructure Facility Centre of D.B.T, Dept of Zoology, D.G (P.G.), College, Kanpur, \\ ${ }^{2}$ Bioinformatics Infrastructure Facility Centre of D.B.T, D.G (P.G.), College, Kanpur, ${ }^{3}$ M.Sc. Biotechnology, \\ D.G.(P.G.), College, Civil Lines, Kanpur
}

\begin{abstract}
Pharmacokinetics and pharmacodynamics modeling is a clinical tool in assistingthe drug development process. The rational for positive clinical testing has been established as pk/pd modeling. Absorption, distribution, metabolism, and excretion (ADME) and chemical reactivity related toxicity is, low while biological receptor activity is higher dimensional in chemistry space and this is partly explainable by evolutionary pressures on ADME to deal with endobiotics and exobiotics. ADME is hard to predict for large data sets because current ADME experimental screens are multi - mechanisms and predictions get worse as more data accumulates. The compounds were analyzed for binding sites. The roots and rhizomes of natural herbs have been used to treat mental weakness, cancer, hypo and hyper thyroidism anti-bacterial disease, viral disease, antifungal, anti-parasitic, oncological disease and also exhibits cardiovascular protective activity and used in the treatment of neural disease. One of the main pharmacological problems today in the treatment of chronic inflammation diseases consists of the fact that anti-inflammatory drugs usually exhibit side effects. The natural products offer a great hope in the identification of bioactive lead compounds and their development into drugs for treating inflammatory diseases computer drug design has proved to be a very useful tool for discovering new drugs. Nature is exhaustible source of natural compounds. The plant possesses immunomodulatory, analgesic, anti convulsant, anti-tubercular, anti- inflammatory, spermicidal, central nervous system, depressive and cardio stimulant properties. The main natural compounds used in cancer therapy and prevention, the historical aspects of their application and pharmacognosy. Clinical trials of marketed formulation showed very encouraging results.
\end{abstract}

Keywords: Pharmacokinetics, Pharmacodynamics, Natural compounds, Endobiotics, Exobiotics

\section{Introduction}

The two most primary questions in drug development process are "has the right drug been selected?" and "has the optimal dosage regimen been established?" Both questions can be addressed using an integrated pharmacokinetics (pk) / pharmacodynamics (pd) approach. One recognized the failure to determine a safe and effective dosage regimen for use in the clear down fall of the clinical trials. Both questions can be addressed using an integrated pharmacokinetics ( $\mathrm{pk}$ ) / pharmacodynamics (pd) approach. One recognized the failure to determine a safe and effective dosage regimen for use in the clear down fall of the clinical trials.(1)While dosage regimens of drugs with narrow therapeutic windows are often adjusted account for $\mathrm{pk} / \mathrm{pd}$ relationship, they are largely ignored for drugs with less obvious dose response relationships. The main impediments to implementation of a pk/pd and toxicokinetics in rational drug development. (2)

Pharmacokinetics is the study of the time course of drug absorption, distribution, metabolism, and excretion (ADME) and how these ADME processes are related to the intensity and time course of the pharmacological (therapeutic and toxic) effect of drugs .(3) If the compounds possess reasonable phsicochemical properties, have low to intermediate clearance and reasonable absorption adequate oral bioavailability may be achieved because conducting an in vivo experiment is time consuming and material consuming, early discovery efforts have focused on assessing in vivo or in situ absorption potential and in vivo metabolic stability.(4)To support clinical development of new chemical entities (NCEs), the three main reasons for clinical failure of an NCE are lack of efficacy, toxicity and unfavorable pharmacokinetic properties. All three are poorly understood and difficult to predict.(5) Several approaches have been described for predicting human pharmacokinetic properties from in vivo preclinical pharmacokinetic data.(6)

India has an ancient heritage of traditional medicines. The ancient medical reports that surgery was performed but physicians also recommended the use of some natural, and especially plant products, which represents interesting point of comparison with current knowledge. Natural products play a relevant role in many diseases. (7) Alternative system of medicines, Ayurveda, siddha, and Chinese medicines has been our single most successful source of medicine. Each plant is like a chemical factory capable of synthesizing a limited number of highly complex and unusual chemical substances derived from plants that are considered as 
important herbs currently use, while several other herbs are simple synthetic modifications of the natural products.

Natural remedy includes use of many herbs, primarily ashvagandha, astragalus, alum, aloe Vera, dandelions, alfalfa, beech drops, cascara, eucalyptus etc. the rhizomes of the herb being over exploited for medicinal use, habitat degradation and other biotic interferences. The rhizome of the herb is considered as the official plant part found to have excellent therapeutic potential. As mentioned in Ayurveda, the roots and the rhizome of natural herbs are used to treat many diseases like viral, antibacterial, cancer, inflammation, antifungal etc.(8)

Natural products traditionally have played an important role in herb discovery and were the basis of the most easily medicines. Over the least 10 to 15 years advances in x-ray crystallography and NMR, and alternative herb discovery methods such as rational herb and combinatorial chemistry have placed great presence upon natural product herb discovery programmes. (9) Herbal mixtures and new use of existing herbs have not been listed exhaustively. During the last few years, natural product based herb discovery is increasing based on new technologies, such as combinatorial synthesis and high throughput screening, and their associated approaches. (10)

Pharmacokinetics and pharmacodynamics models integrated the pharmacokinetic model (relationship between dose and concentration vs. time). Dose - effect predictions are generally achieved by fitting the study data to an appropriate pk/pd model. (11)

Herbs action is mediated by the time course of free herb concentration that is available to bind to a targeted receptor. (12) Pharmacokinetics was intended, in part, to be a tool to optimize the design of biological experiments with herb. Orally delivered pharmacologically active compounds must have favorable absorption and clearance properties and satisfactory metabolic stability to provide adequate systemic exposure to elicit a pharmacodynamics response. (13) High throughput screening (HTS) efforts require rapid methods and the use of minimum amounts of compounds so that new molecules can be synthesized based upon the predicted or experimental outcome of pharmacokinetic and/or toxicity experiments in a timely fashion. (14)

The medicinal benefits of the plant has been valued for centuries in Indian Ayurveda, in Unani in ancient Greek and Arab, and in ancient Egypt and Rome. The use of botanicals plants herbs as medicines predates recorded history and represents the most significant direct antecedents to modern medicines. The currently only about 10,000 herbs like compounds. Herbs like is defined as those compounds that have sufficiently acceptable ADME properties and sufficiently acceptable toxicity properties to survive through the completion of human phase 1 clinical trials.

Antibacterial: - Natural herbs played a pivotal role on the development of antibacterial herbs. Antibacterial herbs improved due to widespread nature of antibacterial resistance sales of new antibacterial may be limited due to potential rapid antibacterial resistance and possibility that a new herb may be quarantined for use only as a least resorts. Some natural herbs are used in antibacterial are barberry, Echinacea, elecampane, golden seal, etc.

Anti-parasitic: -Micro sporidiosisis caused by the spore forming unicellular parasite entero cytosol bieneusi and is of major concern to immune compromised patients in who it causes chronic diarrhea. Fumagillin plays an important role in the treatment of bees with nosema disease, which caused by infection with the protozoan organism. Some anti-parasitic herbs are black berry roots, buck thorn bark, catnip herbs, and myrrh gum etc.

Antifungal: - Most antifungal herbs are used today have some connection to natural herbs. Some herbs are group of naturally occurring lipo peptides produced by various fungi that display potent antifungal acting by inhibition of $1,3-\beta$-D glucan synthesis in the fungal cell wall.

Streptomyces species that display broad spectrum antifungal activity. Their mechanism of action involves complexation with ergostereol and and destabilization of the fungal all membrane, which causes increased membrane permeability and fungal death. Some antifungal herbs are used are as sage herb, etc.

Antiviral:-Some of the greatest health risks known to humans are caused by viral diseases such as HIV, hepatitis B and C, Ebola influenza, dengue fever, and yellow fever. There has been considerable effort over the last 20 years invested into antiviral herbs discovery, especially in the field of HIV. In addition to development of small molecule antiviral herbs, vaccines also are commonly used to try preventing disease like influenza, measles, mumps, polio and small pox. Some herbs are used in anti-viral are as black berry root, barberry, beech drops, cascara bark, cloves fruit etc.

Neurological disease: - Some of the earliest natural herbs derived herbs used for the treatment of pain and central nervous system disease. Some herbs are used in neurological are as lady slipper root, mullein leaf etc. 
Cardiovascular and metabolic disease: - Natural herbs have made a major impact in the treatment of cardiovascular and metabolic diseases. Medicine for the treatment of acute coronary syndromes. It is also being evaluated as an adjunct to cardiovascular intervention such as angioplasty and coronary bypass graft surgery. Some herbs are used in cardiovascular disease are as garlic bulb, alfalfa herb, aloevera, angelica root ginkgo leaf etc.

\section{Immunological, inflammatory}

The discovery that the weak antifungal was an immune suppressant revolutionized organ transplantation and since then, natural herbs have played a pivotal role in the development of most immune suppressive herbs. Anti-inflammatory herbs used in controlling asthma are the cortico steroids. Some herbs are as chicory root, eucalyptus leaf, evening primrose herb, Chamomile flower etc.

Oncological disease: - Natural compounds in clinical development for oncological have been reviewed extensively in the last few years. As consequences, compounds in trials have been tabulated according to their leads compounds source: plant, microorganism and marine. Some herbs are used to cure oncological disease are as bayberry root bark, beech drops, green tea etc.

\section{Tools and techniques}

Database:-

Drug bank: -www.drugbank.ca/ the drug bank database is a unique bioinformatics resource that combined details for natural compounds that is smiles notation, pharmacological properties, targets, toxicity etc.

Pubchem: -www.ncbi.nlm.nih.gov , NCBI > Chemicals \& Bioassays The PubChem Compound Database contains validated chemical depiction information provided to describe substances in PubChem Substance. Structures stored within PubChem Compounds are pre-clustered and cross-referenced by identity and similarity groups.

Chemspider: -www.chemspider.com/ChemSpider is a free chemical structure database providing fast text and structure paste the smile notation and draw structure on page.

\section{Software}

Marvin sketch: -www.chemaxon.com > Products > Marvin an advanced chemical editor for drawing chemical structures, queries and reactions. Its version is 6.0. MarvinSketch allows users to quickly draw molecules through basic functions on the GUI and advanced functionalities such as sprout drawing, customizable shortcuts, abbreviated groups, default and user-defined templates and context-sensitive popup menus.

T.E.S.T (Toxicity Estimation Software Tool)www.epa.gov/nrmrl/std/qsar/qsar.html its version is 4.1 . T.E.S.T has been developed to allow users to easily estimate toxicity using a variety of QSAR methodologies.

Toxicity end points:-T.E.S.T allows you to estimate the value for several toxicity end points

$>96$ hours fathead minnow LC 50 (concentration of the test chemical in water in $\mathrm{mg} / \mathrm{l}$ that causes $50 \%$ of fathead minnow to die after $96 \mathrm{hrs})$.

$>48 \mathrm{hrs}$ daphnia magna LC50 (concentration of the test chemical in water in $\mathrm{mg} / \mathrm{l}$ that causes $50 \%$ of daphnia magna to dies after $48 \mathrm{hrs})$.

$>48$ hrstetrehymenapyriformis IGC50 (concentration of the test chemical in water in $\mathrm{mg} / \mathrm{l}$ that causes $50 \%$ growth inhibition to tetrahymenapyriformis after $48 \mathrm{hrs}$ ).

$>$ Oral rat LD50 (amount of chemical in $\mathrm{mg} / \mathrm{kg}$ body weight that causes $50 \%$ of rats to die after oral ingestion).

$>$ Bioaccumulation factors (ratios of the chemical concentration in fish as a result of absorption via the respiratory surface to that in water at steady state).

$>$ Developmental toxicity (whether or not a chemical causes developmental toxicity effects to humans of animals).

$>$ Ames mutagenicity (a compound is positive for mutagenicity if it induces revertant colony growth in any strain of salmonella typhimurium).

T.E.S.T also allows you estimate several physical properties:-

$>$ Normal boiling point (the temperature in deg.cel. at which a chemical boils at atmospheric pressure).

$>$ Density (the density in $\mathrm{g} / \mathrm{cm}$ cube )

$>$ Flash point (the lowest temperature in deg. Cel. At which it can vaporize to form an ignitable mixture in air).

$>$ Thermal conductivity (the property of a material in units of $\mathrm{mw} / \mathrm{mk}$ reflecting its ability to conduct heat) 
$>$ Viscosity (a measure of the resistance of a fluid to flow in it defines as proportionality constant between shear rate and shear stress).

$>$ Surface tension (a property of the surface in $\mathrm{dym} / \mathrm{cm}$ of a liquid that allows it to resist an external face).

$>$ Water solubility (the amount of a chemical in $\mathrm{mg} / \mathrm{l}$ that will dissolve in liquid water to form a homogenous soloution).

Methodology: Retrieve the natural compound from pubchem and drugbank . Take the mol. , sdf, smile notation of the natural compound from pubchem and drugbank data. Download the TEST software and draw the structure of natural compound on TEST software. Predict the physical properties (normal boiling point, vapour pressure, melting point, flash point, density, surface tension, thermal conductivity and predict the toxicity of the natural compound .

\section{Result and Discussion:}

The primary goal of the project was to unearth natural compounds that can be used as an agonist for the treatment of different diseases that is antiviral, antifungal, antiparastic, cardiovascular, influenza, hepatitis, oncological disease, chronic inflammation, disease etc. Natural compounds isolated from the herbs like bark, leaves, root, stem etc. A total of 80 natural compounds analyzed through T.E.S.T. T.E.S.T (Toxicity Estimation Software Tool) have been developed to allow users to easily estimate toxicity using a variety of QSAR methodologies. By using T.E.S.T software version 4.1 predict the toxicity properties (i.e., Fathead minnow LC50, Daphnia magna, T. pyriformis IGC50, Oral rat LD50, Bioaccumulation factors, developmental toxicity and Mutagenicity.) and physical properties (normal boiling point, vapor pressure, flash point, melting point, water solubility etc.) in natural compounds.

96 hrs. Fathead minnow LC50 is lethal concentration that kills 50\% of the animals in the concentration. In air that kills $50 \%$ of the test animals during the observation periods the LC50 value. In the prediction value of fathead minnow,

The values ranging 10 or less than 10 shows extremely toxic. Compounds such as alfalfa, astragalin, barberry root, bayberry root bark, bee pollen, beta sitosterol, black walnut hull, borage leaf, burdock root, butchers broom, button snake root, chamomile flowers etc. are showing lc50 value in this range. So all of these herbs can be highly toxic in aquatic form.

The values ranging 10-100 is highly toxic the compounds such as alum root, amantadine, black willow bark, blessed thistle herb, buckthorn leaf, cats claw, chicory root etc.The values ranging 100-1000 is moderately toxic the compounds such as like angelica root, beech drops,

48 hrs.Daphnia magna LC50 48 hrs. It is predicted same as in the fathead minnow. It represents the concentration in water which kills half of daphnia magna in $48 \mathrm{hrs}$.

The values ranging 10 or less than 10 alfalfa, alum root, astragalin, bayberry root bark etc.

The values ranging 10-100 is highly toxic the compounds such as amantadine, black willow bark, buckthorn, cats claw etc.

The values ranging 100-1000 is moderately toxic the compounds such as corn silk etc.

Tetra hymenapyriformis IGC50 represents the $50 \%$ growth inhibitory concentration of the t.pyriformis organism after $48 \mathrm{hrs}$. In tetra hymenia pyriformis igc50 is predicted that the value of IGC50 value is more the toxicity of natural compounds is less. It is aquatic toxic.

Astragalin, astragalus, bee pollen, black walnut hull, borage leaf, button snake root, cleavers leaf, elecampane leaf, evening primrose herb, flax seed, kelp flower, marigold flower, myrrh gum, oat straw, parsley leaf, ginger these natural compounds are toxic.

Alfalfa, alum root, angelica root, bay berry root bark, beech drops, black willow bark, blessed thistle herb, buckthorn leaf, burdock root, butchers broom, cats claw, chamomile flowers, corn silk, dandelion root, fennel seed, garlic bulb, golden ragwort, golden seal root, gota kola herb, lady slipper root, licorice root, lily valley root, maidenfair hair herb, maple bark, mayapple root, mullein herb, paudoarco bark, peach tree leaves, peppermint herb, pisessipua leaf, perickley ash bark, turmeric, thyme herb, valerian root, kava kava root these natural compounds are non-toxic.

Amantadine, avocado, barberry root, beta sitosterol, cascara fruit, chicory root, cloves fruit, comfrey root, Echinacea, eucalyptus, fever few herb, gentian root, green tea, kaempferol-4-alpha, lobelia herb, milk thistle herb, mistletoe herb, orange peel, Oregon grape root, passion flower herb, periwinkle herb, pink root, queen of meadow root, horsetail grass herb, rimantadine, hydrangea root, strawberry herb, turkey rhubarb root, juniper berries these natural conpounds could not predicted from T.E.S.T.

Oral rat LD50 in which LD is lethal concentration of 50\% of the test animals during the observation periods.The values ranging 10 or less than in this no compound form. The values ranging 10-100 is highly toxic the compounds such as evening primrose herb etc. 
The values ranging 100-1000 is moderately toxic the compounds such as alfalfa, amantadine, bayberry, bee pollen, beech drops, beta sitosterol, black walnut hull, black willow bark etc. The values ranging 1000-10,000 are slightly toxic the compounds such as alum root, angelica root, astragalin, astragalus, barberry, blessed thistle herb etc. Practically nontoxic 10,000-100,000 the compounds such as flax seed, maidenhair fern herb etc.

Bioaccumulation factors are the ratio of the chemical concentration in biota as a result of absorption via the respiratory surface to that in water at steady state. In bioaccumulation factors the predicted value is $\geq$ (3.5) $\log$

Alfalfa, alum root, amantadine, astragalin, bayberry root bark, beech drops, cats claw, cleavers leaf, corn silk, eucalyptus, kaempferol-4-alpha,orange peel etc. these compounds are not toxic.

Parsley leaf, Oregon grape root, hydrangea root, kava kava root, horsetail grass, queen of meadow root etc. these natural compounds could not predicted from TEST.

Developmentaltoxicity is whether or not a chemical causes developmental toxicity effects in humans and animals. Developmental toxicity includes any effect interfering with normal development, both before and after birth. Their value comes in two form toxicant and non-toxicant.

Toxicant compounds such as:-alfalfa, alum root, amantadine, angelica root, astragalin, astragalus, avocado, barberry root, bayberry root bark, beech drops, beta sitosterol, black walnut hull, blessed thistle herb, borage leaf etc.

Non toxicant compounds such as:-bee pollen, black willow bark, garlic bulb, golden seal root, maidenhair fern herb, maple bark, pisessipua leaf, perickley ash bark, rimantadine, strawberry herb, juniper berries.

The natural compounds which are shows positive value in mutagenicity such as alum.Astragalin, astragalus root, black walnut hull, butchers, cleavers leaf, cloves fruit, comfrey root, Echinacea, kaempferol-4alpha, mistletoe herb, orange peel, Oregon grape rot, horsetail grass herb, strawberry herb, valerian root, kava kava root. The natural compounds which are shows negative values that are leave and which are shows positive values are taken further project work.

Normal boiling point, Flash point, Thermal conductivity, Vapor pressure,Melting point, Water solubility the physical value is predicted.

\section{Conclusions}

The identification and development of natural compounds and their derivatives have greatly contributed to this progress and many of these compounds are now being used in clinical practice. Nature is still today a rich source of active principles against many diseases natural compounds comprise either classical cytotoxic moieties. Newly discovered exotic natural compounds with possibly relevant preventive properties. It is important to emphasize that only rigorous preclinical and clinical studies along with a precise understanding of pharmacology of new compounds may assure the selection of active and safe different disease drugs including natural compounds.

It has been shown in the above result analysis table that there no side effects shown in natural compounds if there is toxicity is found is very minute in that compounds. If the value of natural compounds is positive it will show less lethal toxicity. In 80 natural compounds by use of T.E.S.T (Toxicity Estimation Software Tool) predict the mutagenicity value (shown in above table) and in that prediction it has been shown that there is some natural compounds shows positive.

In conclusion, the application of natural compounds in the treatment of many different diseases, the very common plague of modern times, has resulted in increased therapeutic efficacy. Research results both testify to the evolution of knowledge coming from pharmacognosy and its historical roots in ancient herbal medicines, as well as to the great possibilities of future progress by means of a rational, natural product- based drug discovery approach.

\section{Acknowledgement}

The author wish to thank BIFC, Coordinator Dr.(Mrs.) VIJAY LAXMI SAXENA, For providing constant support and encouragement for research at Bioinformatics Centre.

\section{References}

[1]. Aarons L. Population pharmacokinetics: theory and practice. Br j ClinPharmacol. 1991; 32(6):669-670.

[2]. Bagchi A, OshimaY,Hikino H. validity of oriental medicines . Neollignans and lignans of Nardostachysjatamansi roots. Planta Med $1991 ; 57: 96-97$.

[3]. Gibaldi M, Perrier D: Pharmacokinetics, edn2. Edited by Gilbaldi M, Perrier D. new York: Marcel Dekker, inc; 1982

[4]. Lasagna SE, Naranjo CA, Dose Response Relationship in Clinical Pharmacology. Temple R. Dose response and registration of new drugs. The Netherlands: Elsevier; 1989: 145-167 2005, 22,162-195

[5]. Lave T, Coassolo P, Reigner B: Prediction of hepatic metabolic clearance based on inter species allometric scaling techniques and in vitro - in vivo Correlations, clin Pharmacokinetics 1999, 36: 211-231 
[6]. Mark S. Butler. Natural Products to drugs :natural product derived compounds in Clinical trials

[7]. Regner BG, Williams PE, Patel IH an evalution of the integration of pharmacokinetics and pharmacodynamics principles in clinical drug development: experiences within Hoffman La Roche. ClinPharmacokinet. 1997;33

[8]. Smith DA, vandeWaterbeemed H: Pharmacokinetics and metabolism in early drug discovery , CurrOPinChemBiol 1999, 3: 373 378

[9]. StefaniaNobili, Dontella Lippi, EwaWitort, Martino Donnini ,LetiziaBausi, Enrico Mini, Sergio Capaccioli. Natural compounds for cancer treatment and prevention 2009

[10]. Stewart BM, Wang Y, Surendran N: Ex vivo approaches to predicting Oral Pharmacokinetics in human. Annu Repo Med Chem $2000,35: 299-307$

[11]. Van de Waterbeemed H: Intestinal permeability: prediction from theory. In Oral Drug Absorption:Prediction and Assessment. Edited by Dressman JB, Lennernas H. New York: Marcel Dekker, Inc; 2000:31-49

[12]. Yacobi A, Skelly JP, Shah VP. Rationale for the pharmacokinetics and pharmacodynamics in early drug developmenta. Integration of pharmacokinetics, pharmacodynamics, toxicokinetics in Rationale Drug Development. New York, 1993 Plenum press. 